\title{
Extended Traffic Alert Information to Improve TCAS Performance by means of Causal Models
}

\author{
Jun Tang, ${ }^{1}$ Miquel Angel Piera, ${ }^{1}$ Yunxiang Ling, ${ }^{2,3}$ and Linjun Fan ${ }^{2}$ \\ ${ }^{1}$ Department of Telecommunication and System Engineering, Autonomous University of Barcelona, 08202 Sabadell, Spain \\ ${ }^{2}$ Department of Management Science and Project, Police Officer College of Chinese Armed Police Force, Chengdu 610213, China \\ ${ }^{3}$ Science and Technology on Information Systems Engineering Laboratory, National University of Defense Technology, \\ Changsha 410072, China
}

Correspondence should be addressed to Yunxiang Ling; yunxiang_ling@yeah.net

Received 23 January 2015; Revised 20 May 2015; Accepted 16 June 2015

Academic Editor: Ricardo Aguilar-López

Copyright (C) 2015 Jun Tang et al. This is an open access article distributed under the Creative Commons Attribution License, which permits unrestricted use, distribution, and reproduction in any medium, provided the original work is properly cited.

\begin{abstract}
Near-midair collisions (NMACs) between aircraft have long been a primary safety concern and have incessantly motivated the development of ingenious onboard collision avoidance (CA) systems to reduce collision risk. The Traffic Alert and Collision Avoidance System (TCAS) acts as a proverbially accepted last-resort means to resolve encounters, while it also has been proved to potentially induce a collision in the hectic and congested traffic. This paper aims to improve the TCAS collision avoidance performance by enriching traffic alert information, which strictly fits with present TCAS technological requirements and extends the threat detection considering induced collisions and probabilistic pilot response. The proposed model is specified in coloured Petri net $(\mathrm{CPN})$ formalism, to generate by simulation all the future possible downstream reachable states to enhance the followup decision making of pilots via synthesising relevant information related to collision states. With the complete state space, the potential collision scenarios can be identified together with those manoeuvres that may transform a conflict into a collision. The causal TCAS model is demonstrated to work effectively for complex multiaircraft scenarios and to identify the feasible manoeuvres that contribute to reduce the nonzero TCAS-induced collision risk.
\end{abstract}

\section{Introduction}

The Traffic Alert and Collision Avoidance System (TCAS), which is utilized as the final safeguard to decrease the risk of near-midair collisions (NMACs), is one of the mandatory equipment on all commercial transport aircraft, and its latest version is TCAS II 7.1 [1]. The TCAS equipped in aircraft is an instrument that constitutes a safety network in midair. The main responsibility of TCAS is to notify and avoid collision with any airborne object in a radius distance of $14 \mathrm{NM}$ [1]. TCAS technology does not dominate the vehicle directly; it just issue advisories to crews in corresponding aircraft on how to resolve conflict using vertical manoeuvres. When an intruder approaches in the periphery of preconcerted protected volume of the main aircraft, a threat is detected and the tracking of intruder aircraft is performed via broadcasting inquiries and receiving answers from the intruder's Mode $S$ transponder [2].
TCAS executes independently of ground-based systems, while it is basically supported with relevant surveillance equipment onboard the vehicle. TCAS I and TCAS II (the improved version) differ primarily in the capability of alerting [1]. TCAS I can offer traffic advisories (TAs) to aid the crews in the visual acquisition of nearby aircraft, while TCAS II can supply them with not only TAs but also resolution advisories (RAs).

TCAS only provides a signal of upward or downward advisory as a solution of an emergent threat. In high-density scenarios, the execution of an RA may initiate another new secondary threat (negative domino effect) situation in which the pilots have no enough time to respond [3]. Several encounter models have been previously developed by various organizations to support the estimation of collision risk. In [4], a new cooperative aircraft encounter model is proposed based on the generation of random threats in fast-time Monte Carlo simulations to test the TCAS and evaluate the NMAC 
risk. Authors implemented a dynamic Bayesian network to capture the real-time vertical velocity to simulate the collision avoidance (CA) course. Reference [5] develops the conceptual model based on stochastically and dynamically coloured Petri nets (SDCPN) which has different core elements of TCAS operations. In addition, it completely supports the quantitative analysis of aircraft encounters. The three dimensions (3D) collision risk model (CRM) provided in [6] assesses aircraft safety level based exclusively upon the track process. It is proposed to supplement the accident databases, therefore offering valuable information on impacting factors inferred from the deep assessment of neighbouring scenarios. To generate multithreat encounters, [7] extends the statistical techniques used to develop pairwise correlated encounters. Their study results quantitatively indicate the variation of risk ratio which measures how the probability of an NMAC changes when an aircraft is equipped with TCAS versus being unequipped. Besides, in our previous research $[8,9]$, the causal encounter models have been proposed and improved to identify TCAS weaknesses (potential collision) by predictively generating the flyable scenario for a specific scenario, and the relationship between potential collisions and different pilot delays has been displayed.

However, in the heavy traffic, TCAS may induce a potential collision. In addition, there is a lack of mechanisms to improve the TCAS avoidance performance in dense air traffic. This study relies on a causal CPN model which is absolutely in view of the TCAS-based avoidance operations, to generate all the future possible states in which the potential collision states should be underlined. In this paper, it is considered high-density traffic scenarios with all aircraft TCAS equipped. Recorded radar data show that the crews in corresponding aircraft do not follow the TCAS logic (e.g., not following the TCAS advisory or delayed response manoeuvre) [10]. In terms of practicality, each aircraft involved in the TCAS-TCAS scenario may amend or not amend the heading when an RA is aroused. The main contributions of this research are

(i) the causal TCAS model operates when a TA is declared and aims to provide better advisories at the subsequent RAs. It provides a complete and successive view of state and its evolution; thus, the occurrence process of TCAS-induced collision would be more clearly sensed. A warning will be automatically displayed in the pilot visualization tools at RA when the improper manoeuvres may induce a collision for the current traffic situation,

(ii) the mathematical model for TCAS operations and pilot response are clearly specified in a TCAS-TCAS encounter. For the whole sociotechnical air traffic management (ATM) system, TCAS acts as one of the core parts to ensure flight safety. Obviously, the behaviours (active or passive) of pilots would make an important influence on avoiding collision. Using a series of mathematical equations, this complete CA process including probabilistic pilot response has been conceptually described as the theoretical basis for constructing the encounter model,

(iii) this causal model can enhance the TCAS performance at the operational level in high-density traffic scenarios (without the need to heighten or change the relevant logic) to enable precise monitoring of all of the traffic to assure safe and efficient operations.

Note that the causal TCAS model, which plays an important role in the CA process for multiple aircraft, does not take the horizontal manoeuvring into consideration. It fully abides by the TCAS logic and only determines all of the possible future states that could aid decision making for crews in the involved aircraft. If a combination of horizontal and vertical steering would synchronously be considered, then many potential "fall-out" trajectories could arise. Besides, several legal and technical principles have to be reset by aviation authorities, and the existing TCAS logic should be reconstructed.

The framework of this paper is as follows: Section 2 illustrates the conceptual model of TCAS with the possible pilot reactions; Section 3 represents the CPN-based causal model; Section 4 shows the simulation results and provides the analysis of a case scenario; finally, the conclusions are summarized in Section 5.

\section{Mathematical Description of Avoidance Operations}

This section specifies the conceptual model for improving the CA performance of TCAS. For Aircraft $i(i=1,2, \ldots, n)$, its dynamic characteristics at time $t$ can be described as follows:

$$
\begin{aligned}
p_{t}^{i}=\left[\begin{array}{c}
x_{t}^{i} \\
y_{t}^{i} \\
z_{t}^{i}
\end{array}\right], \\
v_{t}^{i}=\frac{d p_{t}^{i}}{d t}=\left[\begin{array}{c}
v_{t}^{i} \cos \gamma_{t}^{i} \cos \theta_{t}^{i} \\
v_{t}^{i} \cos \gamma_{t}^{i} \sin \theta_{t}^{i} \\
v_{t}^{i} \sin \gamma_{t}^{i}
\end{array}\right]=\left[\begin{array}{c}
v_{t, x}^{i} \\
v_{t, y}^{i} \\
v_{t, z}^{i}
\end{array}\right], \\
0<\theta_{t}^{i}<2 \pi,-\frac{\pi}{2} \leq \gamma_{t}^{i} \leq \frac{\pi}{2} .
\end{aligned}
$$

For Aircraft $i$, the formula defines $p_{t}^{i}$ and $v_{t}^{i}$ as its position and velocity at time $t$ in $3 \mathrm{D}$ aerospace. Here, $\gamma_{t}^{i}$ indicates the pitch angle which represents the orientation of velocity vector $v_{t}^{i}$ in the vertical plane, while $\theta_{t}^{i}$ acts as the course angle which is the orientation of velocity vector $v_{t}^{i}$ in the horizontal plane. Considering the geometry of the encounter between Aircraft $i$ and Aircraft $j$, several important parameters can be calculated as follows [9]: 


$$
\begin{aligned}
& T_{t_{\mathrm{CPA},}, h}^{i j}=\frac{-\sqrt{\left(x_{t}^{i}-x_{t}^{j}\right)^{2}+\left(y_{t}^{i}-y_{t}^{j}\right)^{2}}}{\sqrt{\left(v_{t, x}^{i}-v_{t, x}^{j}\right)^{2}+\left(v_{t, y}^{i}-v_{t, y}^{j}\right)^{2}} \cdot \cos \left(\alpha_{t}^{i j}-\beta_{t}^{i j}\right)}, \\
& \cos \alpha_{t}^{i j}=\arctan \left(\frac{v_{t, x}^{i}-v_{t, x}^{j}}{v_{t, y}^{i}-v_{t, y}^{j}}\right) \text {, } \\
& \cos \beta_{t}^{i j}=\arctan \left(\frac{x_{t}^{i}-x_{t}^{j}}{y_{t}^{i}-y_{t}^{j}}\right) \text {, } \\
& T_{t_{\mathrm{CPA}}, z}^{i j}=-\frac{z_{t}^{i}-z_{t}^{j}}{v_{t, z}^{i}-v_{t, z}^{j}} \\
& D_{t, h}^{i j}=\sqrt{\left(x_{t}^{i}-x_{t}^{j}\right)^{2}+\left(y_{t}^{i}-y_{t}^{j}\right)^{2}} \\
& D_{t, z}^{i j}=\left|z_{t}^{i}-z_{t}^{j}\right| \text {, } \\
& D_{t_{\mathrm{CPA},}, h}^{i j}=\sqrt{\left[\left(x_{t}^{i}+v_{t, x}^{i} \cdot T_{t_{\mathrm{CPA}}, z}^{i j}\right)^{2}-\left(x_{t}^{j}+v_{t, x}^{j} \cdot T_{t_{\mathrm{CPA}}, z}^{i j}\right)^{2}\right]+\left[\left(y_{t}^{i}+v_{t, y}^{i} \cdot T_{t_{\mathrm{CPA}}, z}^{i j}\right)^{2}-\left(y_{t}^{j}+v_{t, y}^{j} \cdot T_{t_{\mathrm{CPA}}, z}^{i j}\right)^{2}\right]}, \\
& D_{t_{\mathrm{CPA}}, z}^{i j}=\sqrt{\left[\left(z_{t}^{i}+v_{t, z}^{i} \cdot T_{t_{\mathrm{CPA}}, h}^{i j}\right)^{2}-\left(z_{t}^{j}+v_{t, z}^{j} \cdot T_{t_{\mathrm{CPA}}, h}^{i j}\right)^{2}\right]} .
\end{aligned}
$$

Note that $T_{t_{\mathrm{CPA}}, h}^{i j}$ (horizontal plane) and $T_{t_{\mathrm{CPA}}, z}^{i j}$ (vertical plane) are separately the time to the closest point of approach (CPA). Besides, $\alpha_{t}^{i j}$ is defined as the direction angle of the velocity difference vector while $\beta_{t}^{i j}\left(\alpha_{t}^{i j}-\beta_{t}^{i j} \neq \pm \pi / 2\right)$ indicates the direction angle of the position difference vector. $D_{t, h}^{i j}$ and $D_{t, z}^{i j}$ indicate their distances in the horizontal and vertical planes at time $t . D_{t, h}^{i j}$ and $D_{t, z}^{i j}$ indicate their horizontal and vertical distances at CPA.

To improve the computational efficiency, only the TA which would deteriorate into RA is considered in this research. The situation should meet the following set of conditions:

$$
\begin{aligned}
& \left(0<T_{t_{\mathrm{CPA},},}^{i j}<\mathrm{Time}_{\mathrm{TA}}\right) \wedge\left(0<T_{t_{\mathrm{CPA}}, z}^{i j}<\mathrm{Time}_{\mathrm{TA}}\right) \\
& \wedge\left(D_{t_{\mathrm{CPA}}, h}^{i j}<\mathrm{DMOD}_{\mathrm{RA}}\right) \wedge\left(D_{t_{\mathrm{CPA}}, z}^{i j}<\mathrm{ZTHR}_{\mathrm{RA}}\right) .
\end{aligned}
$$

An RA would be issued when

$$
\begin{aligned}
& {\left[\left(0<\mathrm{T}_{t, h}^{i j}<\text { Time }_{\mathrm{RA}}\right) \wedge\left(0<\mathrm{T}_{t, z}^{i j}<\text { Time }_{\mathrm{RA}}\right)\right]} \\
& \quad \vee\left[\left(D_{t, h}^{i j}<\mathrm{DMOD}_{\mathrm{RA}}\right) \wedge\left(D_{t, z}^{i j}<\mathrm{ZTHR}_{\mathrm{RA}}\right)\right],
\end{aligned}
$$

where Time RA $[1]$ indicates the time to the CPA constraint, while $\mathrm{DMOD}_{\mathrm{RA}}$ and $\mathrm{ZTHR}_{\mathrm{RA}}[1]$ are separately the range and altitude threshold. When an RA occurs, a two-step process is required to generate the appropriate advisories for resolving conflicts [1]. First, the amending sense is obtained on account of the predictive CPA altitude. In terms of practicality, each aircraft has three choices (climb/unchanged/descend) when an RA is aroused. Define $C_{t_{\mathrm{RA}}}^{i}$ as the possible selection of Aircraft $i$ at $t_{\mathrm{RA}}$ :

$$
C_{t_{\mathrm{RA}}^{i}}^{i}= \begin{cases}1, & \text { climb, } \\ 0, & \text { unchanged, } \\ -1, & \text { descend. }\end{cases}
$$

For Aircraft $i$ and Aircraft $j$ which are involved in the same threat, their response combination $C_{t_{\mathrm{RA}}}^{i j}=\left(C_{t_{\mathrm{RA}}}^{i}, C_{t_{\mathrm{RA}}}^{j}\right)$ can be $(1,-1),(1,0),(0,-1),(0,1),(-1,0)$, and $(-1,1)$.

Second, the RA strength is calculated to provide adequate separation between the involved aircraft at CPA. Normally, the initial $a_{0}=0.25 \mathrm{~g}$ acceleration manoeuvre would be adopted within 5 seconds [1]:

$$
\begin{aligned}
& z_{t_{\mathrm{CPA}}, h}^{i}=z_{t}^{i}+v_{t, z}^{i} \cdot T_{t_{\mathrm{CPA}}, h}^{i j}, \\
& z_{t_{\mathrm{CPA}, h}}^{j}=z_{t}^{j}+v_{t, z}^{j} \cdot T_{t_{\mathrm{CPA}}, h}^{i j} .
\end{aligned}
$$

If $C_{t_{\mathrm{RA}}}^{i j}=(1,0)$, for Aircraft $i$, the appropriate amendment strength should be

$$
\Delta_{t, z}^{i}=\operatorname{ALIM}_{\mathrm{RA}}+z_{t_{\mathrm{CPA}, h}^{i}}^{i}-z_{t_{\mathrm{CP}, h}^{j}}^{j} .
$$


Additionally, the acceleration time $t_{a_{0}}^{i}$ of Aircraft $i$ meets the following formula:

$$
\Delta_{t, z}^{i}=\frac{a_{0} t_{a_{0}}^{2^{2}}}{2}+a_{0} t_{a_{0}}^{i}\left(T_{t_{\mathrm{CP}}, h}^{i j}-t_{a_{0}}^{i}\right) .
$$

Thus,

$$
t_{a_{0}}^{i}=T_{t_{\mathrm{CPA}}, h}^{i j}-\sqrt{T_{t_{\mathrm{CPA}}, h}^{i j}-\frac{2 \Delta_{t, z}^{i}}{a_{0}}} .
$$

For the other possible response combinations, the calculation process is similar.

TCAS may induce a domino threat in high-density air traffic. For the secondary threat, pilot response with $a_{1}=$ $0.35 \mathrm{~g}$ acceleration is set within 2.5 seconds [1]. But a domino conflict would deteriorate into an induced collision if the time is not adequate. Assume that Aircraft $i$ has a secondary threat with Aircraft $j$. If at moment $t_{a_{1}}$ the diameter and height of the collision volumes of both aircraft overlap, a potential collision would emerge:

$$
\begin{aligned}
& D_{t_{a_{1}}, h}^{i k}=\sqrt{\left(x_{t_{a_{1}}}^{i}-x_{t_{a_{1}}}^{k}\right)^{2}+\left(y_{t_{a_{1}}}^{i}-y_{t_{a_{1}}}^{k}\right)^{2}} \leq D_{\mathrm{cl}}, \\
& D_{t_{a_{1}}, z}^{i k}=\left|z_{t_{a_{1}}}^{i}-z_{t_{a_{1}}}^{k}\right| \leq H_{\mathrm{cl}} .
\end{aligned}
$$

Here, at time $t_{a_{1}}, D_{t_{a_{1}}, h}^{i k}$ and $D_{t_{a_{1}}, z}^{i k}$ separately indicate the horizontal and vertical distances between the corresponding aircraft. In this paper, a vehicle is modeled as a unique point [3] that is surrounded by a 3D safety volume (cylinder), and therefore a collision will break out when the diameter and height criteria $\left(D_{\mathrm{cl}}\right.$ and $\left.H_{\mathrm{cl}}\right)$ are violated simultaneously.

Because of the possible selections (climb/unchanged/ descend) of each aircraft when an RA fires, there would be a great deal of future states that a scenario could emerge from current state in a multiaircraft scenario.

\section{Causal Analysis Model in CPN Formalism}

In this section, the causal analysis model to improve the TCAS avoidance performance is modelled discretely to consider the continuous dynamics. Based on CPN [11], it aims to generate all the future possible states and screen out the potential collision states that should be forcibly avoided.

3.1. Coloured Petri Net. CPN is an effective modelling formalism for multiple complex systems and it has been widely utilized to realize and verify systems, representing not only the static behaviour and system dynamics but also the information flow [12]. CPN is used as a graphical language which combines the capabilities of a Petri net (PN) [13] with the advantages of an effective programming language, modelling concurrent systems and analysing their properties.

Definition 1. A nonhierarchical CPN is a nine-tuple [14]:

$$
\mathrm{CPN}=(\Sigma, P, T, A, N, C, G, E, I),
$$

where

(i) $\Sigma=\left\{C 1, C 2, \ldots, C_{n c}\right\}$ represent the finite set of colours,

(ii) $P=\left\{P 1, P 2, \ldots, P_{\mathrm{np}}\right\}$ represent the finite set of place nodes,

(iii) $T=\left\{T 1, T 2, \ldots, T_{\text {nt }}\right\}$ represent the set of transition nodes, $P \cap T=\varnothing$,

(iv) $A=\left\{A 1, A 2, \ldots, A_{\text {na }}\right\}$ represent the directed arc set, $A \subseteq P \times T \cup T \times P$,

(v) $N=$ the node function $N\left(A_{i}\right)$, related with the input and output arcs,

(vi) $C=$ the colour set functions $C\left(P_{i}\right)$, specifying the combination of colours for each place $P \rightarrow \Sigma$,

(vii) $G=$ the guard function $G\left(T_{i}\right)$, which is associated with transition nodes and normally used to inhibit the event related with the transition upon the attribute values of the processed entities,

(viii) $E=$ the arc expressions $E\left(A_{i}\right)$, which specify the quantity and type of entities that can be selected among the ones present in the place node to enable the transition,

(ix) $I=$ the initialization function $I\left(P_{i}\right)$, allowing the value specification for the initial entities in the places.

Note that the state space analysis tool $[15,16]$ of CPN can be used to explore the systems' dynamic and to represent all the reachable future state. It is depicted by means of the reachability tree (also called occurrence graph).

Figure 1 illustrates the first level of a reachability tree, and the state vector of the transition (T4 of the causal encounter model described in Figure 2) with 3 places is represented. In each position of the vector, the tokens and their colours that are stored in each place node are represented. Given this initial marking $\left(P 10: 1^{\prime}(5), P 11\right.$ : $1^{\prime}(1,0)+1^{\prime}(1,-1)+1^{\prime}(0,1)+1^{\prime}(0,-1)+1^{\prime}(-1,0)+$ $\left.1^{\prime}(-1,1)\right)$, the only enabled events are those indicated by T4. In addition, $T 4$ has six different combinations of tokens: $1^{\prime}(1,0)$ : climb/unchanged; $1^{\prime}(1,-1)$ : climb/descend; $1^{\prime}(0,1)$ : unchanged/climb; $1^{\prime}(0,-1)$ : unchanged/descend; $1^{\prime}(-1,0)$ : descend/unchanged; $1^{\prime}(-1,1)$ : descend/climb. The result of a transition corresponds to a new state vector (i.e., a new traffic scenario). And therefore CPN model can generate the whole state space of a complex system without any extra behaviour in the simulation [17].

The reachability tree of the CA operations applied to a certain scenario allows us to comprehend the cause-effect relationship of each system action and how the effects are spread downstream. In essence, the analysis problem becomes a search problem in which the ATM can clearly determine the aircraft manoeuvres that could lead to potential collisions and provide feasible (or even optimal) advisories to the involved pilots. It is used as a causal analysis model to improve the TCAS avoidance performance in dense air traffic.

3.2. Causal TCAS Model. Abundant research of TCAS weaknesses explains that, even with TCAS onboard, an induced 


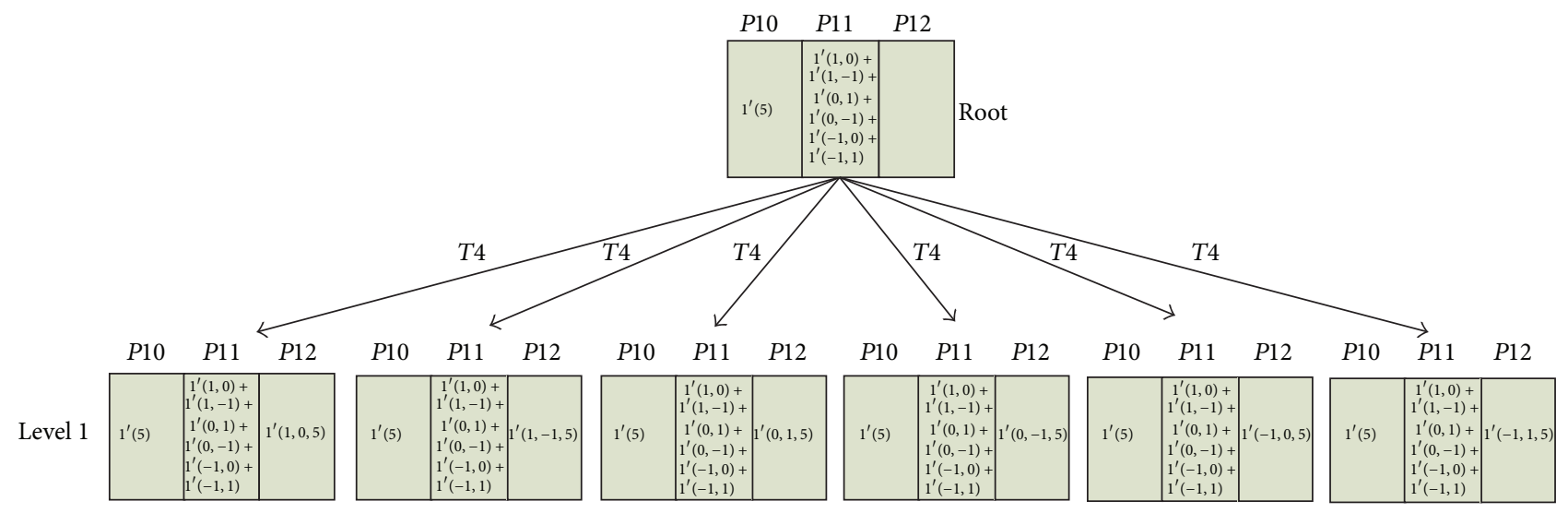

FIgURE 1: An example of reachability tree.

collision also could emerge especially in the dense air traffic [2-9]. Therefore, based on the mathematical model presented in the previous section, a causal analysis model in CPN formalism is critical for the CA process of multiple aircraft. Note that it is complementary to the widely used TCAS smart changes in its logic.

The proposed CPN model considers situations that occur in a short period of time with a local scope, which results in the rectilinear motion of the aircraft from a simplified view. Besides, the local airspace that the multiple aircraft are involved in can be modelled as a Euclidean 3D space. A Cartesian coordinate system [2] is used to represent the planar projection of the Earth with minimum distortion [3]. If the initial positions and speeds of the involved aircraft are known, the sequence of following waypoints forming the partial trajectories in local airspace can be easily calculated.

The proposed CPN model has been depicted using fourteen colours, twenty-three places, and ten transitions. Figure 2 illustrates the causal analysis model described in CPN, and it includes three kinds of agents (Agent TCAS processor, Agent predictive computation, and Agent aircraft crew) that are modelled for the causal analysis.

3.2.1. Agent TCAS Processor. As is depicted in Figure 2, the Agent TCAS processor contains the part of carrying out the TCAS operations. This agent contains three transitions:

(i) T1: Sensitivity Level (SL) [1] Evaluation. Different SLs correspond to different TA and RA thresholds, and SL is determined based on their flight altitudes.

(ii) T2: Threat Detection. The TA is issued when another aircraft approaches and a collision would emerge within 20-48 s (variables are provided in P5) on account of the SL. It tries to draw the pilot's attention and calculates the CPA to inform Agent predictive computation.

(iii) T3: Threat Resolution. TCAS issues the RA when a collision would emerge within 15-35s (variables are provided in $P 7$ ) that is depending on the SL. With the communication of Agent aircraft crew, the RA could be active (climb and descend) or passive (not climb and not descend). In order to explore the possible future situations, this transition also serves to transmit RA waypoint information to the Agent predictive computation.

When the encounter has been resolved, "Clear of Conflict" (CoC) would be advised and the relevant information is stored in $P 9$.

3.2.2. Agent Predictive Computation. This Agent predictive computation contains the part of exploring the complete state space of the possible future situations. This agent integrates with Agent TCAS processor to obtain the related aircraft state which are in the respective CA process and Agent aircraft crew to receive the impact of pilot behaviour. This agent contains six transitions:

(i) T5: Neighbouring Threat Selection. The neighbouring threats would have an interrelationship that may lead to a new secondary conflict or even potential collision. Thus, distance and time tests are implemented on the neighbouring threats (e.g., threat 1 between Aircraft $i$ and Aircraft $j$, threat 2 between Aircraft $k$ and Aircraft l):

$D_{t_{\mathrm{CPA}}}^{12}$

$=\sqrt{\left(x_{t_{\mathrm{CPA}}}^{1}-x_{t_{\mathrm{CPA}}}^{2}\right)^{2}+\left(y_{t_{\mathrm{CPA}}}^{1}-y_{t_{\mathrm{CPA}}}^{2}\right)^{2}+\left(z_{t_{\mathrm{CPA}}}^{1}-z_{t_{\mathrm{CPA}}}^{2}\right)^{2}}$

$\leq D_{\text {narby }}$

$T_{t_{\mathrm{CPA}}}^{12}=\left|t_{t_{\mathrm{CPA}}}^{1}-t_{t_{\mathrm{CPA}}}^{2}\right| \leq T_{\text {narby }}$.

If the distance $\left(D_{t_{\mathrm{CPA}}}^{12}\right)$ and time $\left(T_{t_{\mathrm{CPA}}}^{12}\right)$ at CPA of both threats (threat 1 and threat 2 ) meet the dimension criteria $\left(D_{\text {narby }}\right)$ and time threshold $\left(T_{\text {narby }}\right)$, the relationship is declared to be adjacent.

(ii) T6: Resolution Waypoints Summary. This transition consolidates the multiaircraft waypoints at the beginning of RA and records the pilot responses to advisories for the successive research of domino threat. 


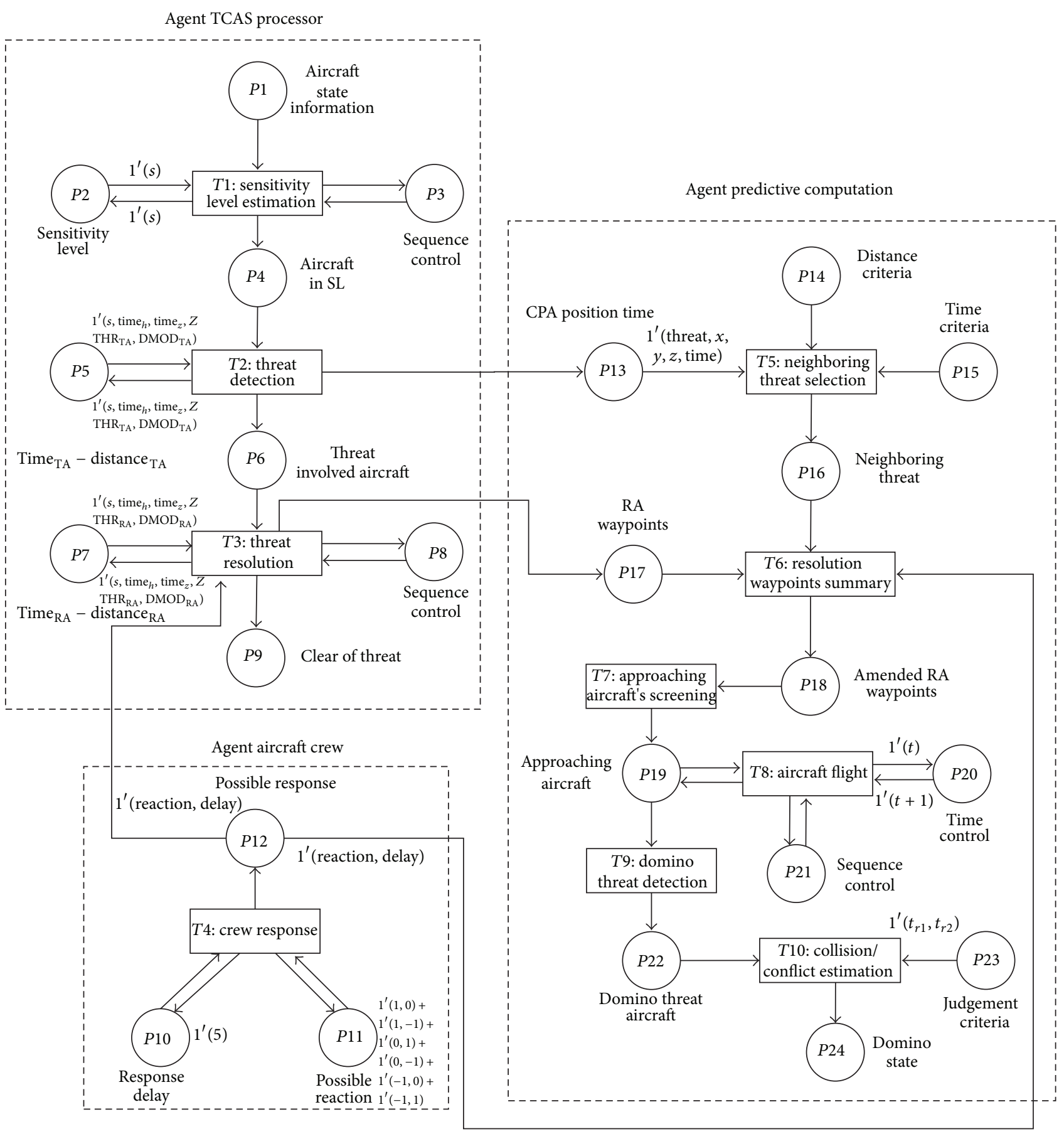

FIgURe 2: Causal analysis model in CPN.

$P 16$ records the neighbouring threats while $P 17$ keeps the amended waypoint of each aircraft to resolve corresponding threat; thus, the aircraft involved in nearby threats which may initiate domino effects are summarized in $P 18$ via T6.

(iii) T7: Approaching Aircraft Screening. To improve the computational efficiency, only the approaching aircraft which are resolving their separate primary threat should be screened out for the secondary threat detection:

$$
D_{t_{\mathrm{RA}}+1}^{i k}-D_{t_{\mathrm{RA}}}^{i k}<0 \text {. }
$$

If the distances $\left(D_{t_{\mathrm{RA}}}^{i k}\right)$ between Aircraft $i$ and Aircraft $k$ are getting smaller, it means that they may encounter with each other.

(iv) T8: Aircraft Flight. Operating this transition one time indicates that the aircraft fly to the next waypoints, 
TABLE 1: Parameter values for the scenarios.

\begin{tabular}{lccccccc}
\hline Equipment & $\begin{array}{c}\text { Detection range } \\
(\mathrm{Nm})\end{array}$ & $\begin{array}{c}\text { RA acceleration } \\
(\mathrm{g})\end{array}$ & $\begin{array}{c}D_{\mathrm{cl}} \\
(\mathrm{NM})\end{array}$ & $\begin{array}{c}H_{\mathrm{cl}} \\
(\mathrm{ft})\end{array}$ & $\begin{array}{c}\text { Subsequent RA acceleration } \\
(\mathrm{g})\end{array}$ & $\begin{array}{c}\text { SL } \\
\text { response time } \\
(\mathrm{s})\end{array}$ \\
\hline TCAS II & 40 & 0.25 & 0.044 & 78.44 & 0.35 & 6 \\
\hline
\end{tabular}

until all threats are resolved or a new secondary threat is detected (T9).

(v) T9: Domino Threat Detection. This transition is similar to $T 2$, while the difference is that the involved aircraft have been in the process of resolving their own primary threat.

(vi) T10: Collision/Conflict Estimation. This transition determines whether the new secondary threat could be resolved or not. The pilots have to resolve the emergent threat, if $T_{t_{\mathrm{CPA}}, h}^{i k}$ and $T_{t_{\mathrm{CPA}}, z}^{i k}$ are smaller than a given time value $T_{\mathrm{cl}}$, and induced collision would emerge as the consequence of previous decisions.

In summary, the Agent predictive computation is ingeniously designed to narrow the expanded state space based on those transitions such as $T 5$ and $T 7$, which can play an important role in dealing with the complex multiaircraft scenarios (e.g., flocks).

3.2.3. Agent Aircraft Crew. This Agent contains the part of representing a core pilot entity in the collision avoidance. This agent includes only one transition $T 4$ to provide probabilistic pilot response, and it connects with two places $P 10$ and $P 11$ in which the crew can be (a) active, the pilot follows the advisories, (b) passive, the pilot would not follow the advisories.

\section{Results}

The simulation experiments are carried out to evaluate the efficacy of the proposed causal analysis model for the improvement of the TCAS avoidance performance. The computational results represent feasible collision-free manoeuvres for multiple aircraft that are modelled with detailed dynamics.

To verify the feasibility of the proposed technique, several simulation experiments have been performed on a multithreat scenario, illustrated in Figure 3. This scenario includes the following characteristics:

(1) It is a fairly complex scenario with multiple aircraft. Indeed, among the first two vehicles, Aircraft 1 have a threat with Aircraft 2; moreover, an encounter emerges between Aircraft 3 and Aircraft 4 which further increases the complexity of the scenario.

(2) It involves the interrelationship (also called domino effects) between neighboring threats. For instance, a new secondary threat may emerge in the process of resolving their respective previous threat.

(3) It allows each involved aircraft to select different heading changes (climb/unchanged/descend).

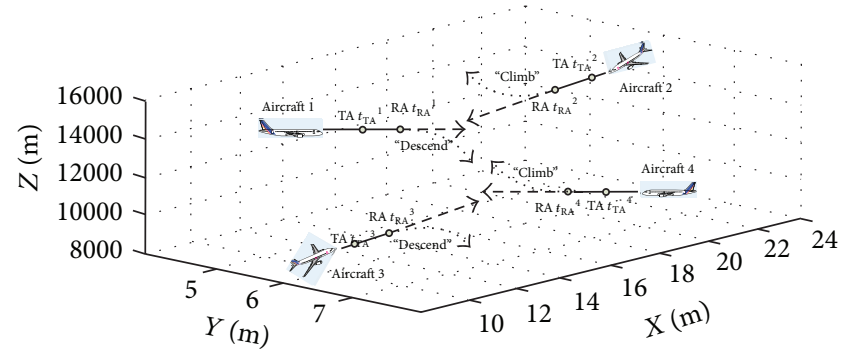

FIGURE 3: Four-aircraft scenario with two nearby threats.

The original TCAS logic is deterministic and the variability in pilot response is not considered.

Table 1 summarizes the parameter values $[1,18]$ utilized in the case scenario that are used to test the feasibility of the proposed model.

RADIUS is used as the state space analysis tool, and it has been shown to be effective for the performance analysis of very demanding and flexible industrial systems in $[19,20]$.

4.1. Deterministic TCAS Logic. This section represents the simulation results of the specific scenario in which the TCAS logic is deterministic and the pilots rigorously follow the advisories. In Figure 3 , variable $t_{\mathrm{TA}}^{i}(i=1,2,3,4)$ is the TA emergence time while $t_{\mathrm{RA}}^{i}(i=1,2,3,4)$ is used for the RA. In the beginning, Aircraft 1 is cruising at FL180 while the nearby Aircraft 2 is descending from FL190. When Aircraft 2 is approaching, a TA occurs. Once the situation deteriorates and an RA is issued, TCAS will fire an advisory that Aircraft 1 descends while Aircraft 2 amends upward to achieve the safe separation. At the same time, the climbing Aircraft 3 encounters the cruising Aircraft 4, and the pilot in Aircraft 3 responds to the downward advisory while Aircraft 4 amends upward. However, an emergent encounter between Aircraft 4 and Aircraft 1 as the domino effect appears, and they have to address the sequent threat. Unfortunately, the left time is not enough for the pilot reaction and aircraft performance. A potential collision exists in the complex scenario when all the involved aircraft absolutely follow the deterministic TCAS logic.

At 7:24:00, the states $1^{\prime}$ (Aircraft, $\left.x, y, z, v x, v y, v z\right)$ of the four aircraft separately are $1^{\prime}(1,13.28,18.40,18000.00,0.18$, $0,0)+1^{\prime}(2,33.16,15.99,18810.00,-0.1,0.1,-10)+1^{\prime}(3$, $12.23,24.27,16880.00,0.08,-0.08,15)+1^{\prime}(4,32.67,18.43$, $17880.00,-0.2,0,0)$. For threat 1 , the TA is issued at 7:24:26 and the RA is issued to ask the pilots in both aircraft to resolve this encounter at 7:24:41. For threat 2, the TA emerges at $7: 24: 28$, and the RA is issued at 7:24:43. Table 2 represents the waypoints from the time of 7:24:46 when Aircraft 1 and Aircraft 2 begin to amend their trajectories. At 7:24:51, there 
TABLE 2: Partial waypoints of the four aircraft.

\begin{tabular}{llcccccccc}
\hline Time & Aircraft & $X(\mathrm{Nm})$ & $Y(\mathrm{Nm})$ & $Z(\mathrm{ft})$ & Time & Aircraft & $X(\mathrm{Nm})$ & $Y(\mathrm{Nm})$ & $Z(\mathrm{ft})$ \\
\hline 7:24:46 & Aircraft 1 & 21.56 & 18.40 & 18000.00 & $7: 24: 49$ & Aircraft 1 & 22.10 & 18.40 & 17985.00 \\
7:24:46 & Aircraft 2 & 28.56 & 20.59 & 18350.00 & $7: 24: 49$ & Aircraft 2 & 28.26 & 20.89 & 18335.00 \\
7:24:46 & Aircraft 3 & 15.91 & 20.59 & 17580.00 & $7: 24: 49$ & Aircraft 3 & 16.15 & 20.35 & 17603.83 \\
7:24:46 & Aircraft 4 & 23.47 & 18.43 & 17880.00 & $7: 24: 49$ & Aircraft 4 & 22.87 & 18.43 & 17886.17 \\
7:24:47 & Aircraft 1 & 21.74 & 18.40 & 17995.00 & $7: 24: 50$ & Aircraft 1 & 22.28 & 18.40 & 17980.00 \\
7:24:47 & Aircraft 2 & 28.46 & 20.69 & 18345.00 & $7: 24: 50$ & Aircraft 2 & 28.16 & 20.99 & 18330.00 \\
7:24:47 & Aircraft 3 & 15.99 & 20.51 & 17590.00 & $7: 24: 50$ & Aircraft 3 & 16.23 & 20.27 & 17607.66 \\
7:24:47 & Aircraft 4 & 23.27 & 18.43 & 17880.00 & $7: 24: 50$ & Aircraft 4 & 22.67 & 18.43 & 17892.34 \\
7:24:48 & Aircraft 1 & 21.92 & 18.40 & 17990.00 & $7: 24: 51$ & Aircraft 1 & 22.46 & 18.40 & 17975.00 \\
$7: 24: 48$ & Aircraft 2 & 28.36 & 20.79 & 18340.00 & $7: 24: 51$ & Aircraft 2 & 28.06 & 21.09 & 18325.00 \\
7:24:48 & Aircraft 3 & 16.07 & 20.43 & 17600.00 & $7: 24: 51$ & Aircraft 3 & 16.31 & 20.19 & 17611.49 \\
7:24:48 & Aircraft 4 & 23.07 & 18.43 & 17880.00 & $7: 24: 51$ & Aircraft 4 & 22.47 & 18.43 & 17898.51 \\
\hline
\end{tabular}

would be a collision between Aircraft 1 and Aircraft 4; the horizontal distance is $\sqrt{(22.46-22.47)^{2}+(18.40-18.43)^{2}}=$ $0.032<0.044 \mathrm{Nm}$ while the altitude interval is $|17975.00-17898.51|=76.49<78.44 \mathrm{ft}$.

Note that the above case scenario of potential collision has been validated by the Interactive Collision Avoidance Simulator (InCAS) [5]. The illustrated case scenario is actually crowded to represent the computational complexity of this proposed causal encounter model:

(1) According to [7], multiaircraft scenarios with only three aircraft realistically comprise over $95 \%$ by analysing the distribution over the number of aircraft involved in a multithreat scenario, based on the radar data from Federal Aviation Administration (FAA) and Department of Defense sites throughout the United States. Thus, the analysed encounter scenario considers four aircraft which is a very rare and saturated scenario in the actual ATM system.

(2) Based on [1], "TCAS II was designed to operate in traffic densities of up to 0.3 aircraft per square nautical mile (NM), that is, 24 aircraft within a $5 \mathrm{NM}$ radius, which was the highest traffic density envisioned over the next 20 years." The proposed scenario considers 4 aircraft corresponding to a density of approximate 0.31 aircraft per square NM that is similar to the highest density in which TCAS could work but could not efficiently resolve all threats.

(3) Although there would be a large number of aircraft in the entire airspace, the aircraft number to be considered by TCAS is limited to a restricted range of the own aircraft. A highly efficient method for avoiding computational redundancies in the state space is clustering, which reduces the general problem to several sets of independent scenarios (known as clusters [8], just as the four-aircraft scenario in this paper) in a partial scope. It means that there may be several aircraft as the surrounding traffic of the four aircraft, but they have been ignored because they have no direct influence on TCAS performance neither in the cluster results.

4.2. Causal Analysis. This section presents the reachability states of this four-aircraft scenario (displayed in Figure 4) that is generated by our causal CPN model. The causal TCAS model begins to operate when both the adjacent and the interactional TAs are declared. For Aircraft 1 and Aircraft 2 which are involved in threat 1 , they separately have three choices (climb/unchanged/descend) when the RA is issued. Evidently, the same heading selections should be avoided. Therefore, their response combination $C_{t_{\mathrm{RA}}}^{12}=\left(C_{t_{\mathrm{RA}}}^{1}, C_{t_{\mathrm{RA}}}^{2}\right) \mathrm{can}$ be $(1,-1),(1,0),(0,-1),(0,1),(-1,0)$, and $(-1,1)$, as shown in the first level of this reachability tree (also illustrated in Figure 1). Immediately afterwards, Aircraft 3 and Aircraft 4 have to resolve the neighbouring threat 2. Analogously, their possible reaction $C_{t_{\mathrm{RA}}}^{34}=\left(C_{t_{\mathrm{RA}}}^{3}, C_{t_{\mathrm{RA}}}^{4}\right)$ also has six options. In view of the overall situation, there would be $6 \times 6=36$ states in Level 2 for the four-aircraft response combination $C_{t_{\mathrm{RA}}}^{1234}=$ $\left(C_{t_{\mathrm{RA}}}^{1}, C_{t_{\mathrm{RA}}}^{2}, C_{t_{\mathrm{RA}}}^{3}, C_{t_{\mathrm{RA}}}^{4}\right)$. The two nearby threats may produce domino effects that would initiate a new secondary encounter or even a potential collision. The tests of domino effect are implemented on the involved aircraft, and five response combinations $(1,-1,1,0),(0,-1,1,-1),(-1,0,-1,1),(-1,1,0,1)$, and $(-1,1,-1,1)$ containing domino threats have been detected. In addition, the conflict would deteriorate into a collision when $C_{t_{\mathrm{RA}}}^{1234}=(-1,1,-1,1)$ and it has been validated and presented in the above section. The graphical illustrations and descriptions of these five scenarios which contain negative domino effect are shown in Table 3.

The state space of this case scenario is generated in $4.7 \mathrm{~s}$, which is significantly less than the time interval between TA and RA of each first encounter (normally $15 \mathrm{~s}$ ). Thus, the proposed CPN model can be employed as the causal CPN model to generate quantitatively the state space of a real-time traffic scenario. A warning can be automatically issued in the pilot visualization tools when the current traffic matches one of the identified collision scenarios. The reachable states without domino conflicts (excluding 5 cases in this scenario) will be recommended for the decision support of pilots. 
TABLE 3: Reachable scenarios with negative domino effect.

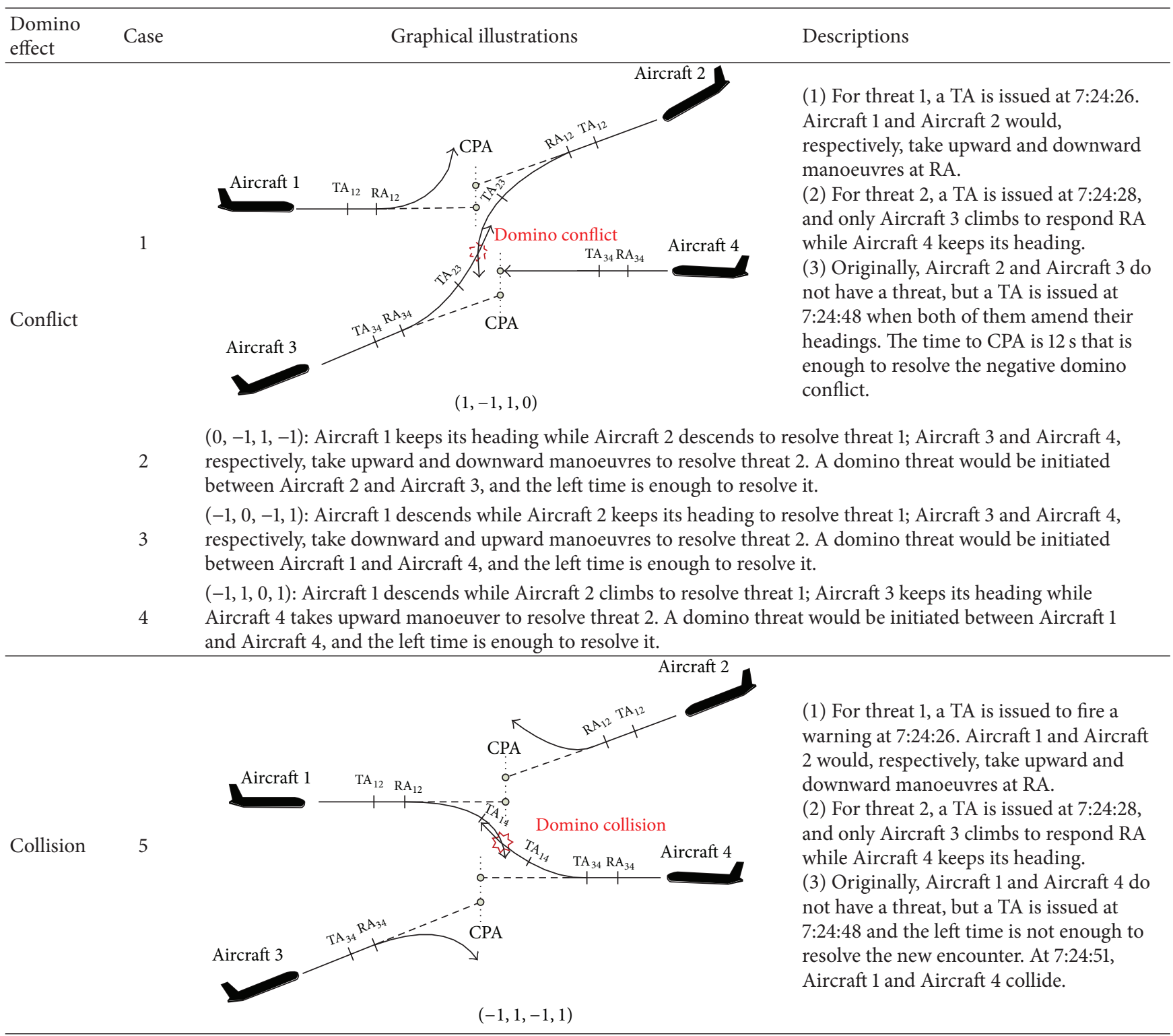

\section{Conclusion}

TCAS has been reflected to greatly improve safety in resolving conflicts. It constantly receives the data such as the altitude, heading, speed, and other discrete inputs from nearby aircraft. These replies are used to compute relative altitude, collision course, and the CPA when compared to the intruders. The overextending aircraft usage for civil and military applications would lead to a higher number of threats which can raise the risk of potential collisions. However, there is a lack of innovative strategies to improve the CA performance of TCAS in multiaircraft scenarios.

The main contribution of this paper is an innovative improvement of the TCAS avoidance performance using a causal CPN model, which is based on the proposed mathematical model for TCAS operations considering pilot reactions. The proposed causal model is absolutely in view of the TCAS-based avoidance operations and considers probabilistic pilot response. Based on the generated state space, the model not only provides a better comprehension of the potential collision occurrences for risk assessment by representing the cause-effect relationship of each action but also aids the crews in the involved aircraft to make a cooperative and feasible option. Besides, several techniques (e.g., eliminating the situations that the aircraft are separate) are utilized to improve the computational efficiency and inventively resolved the problem of expansive state exploration. The causal model can play a major role for resolving TCAS-TCAS encounters in the aircraft flocks and support the development of new TCAS version. 


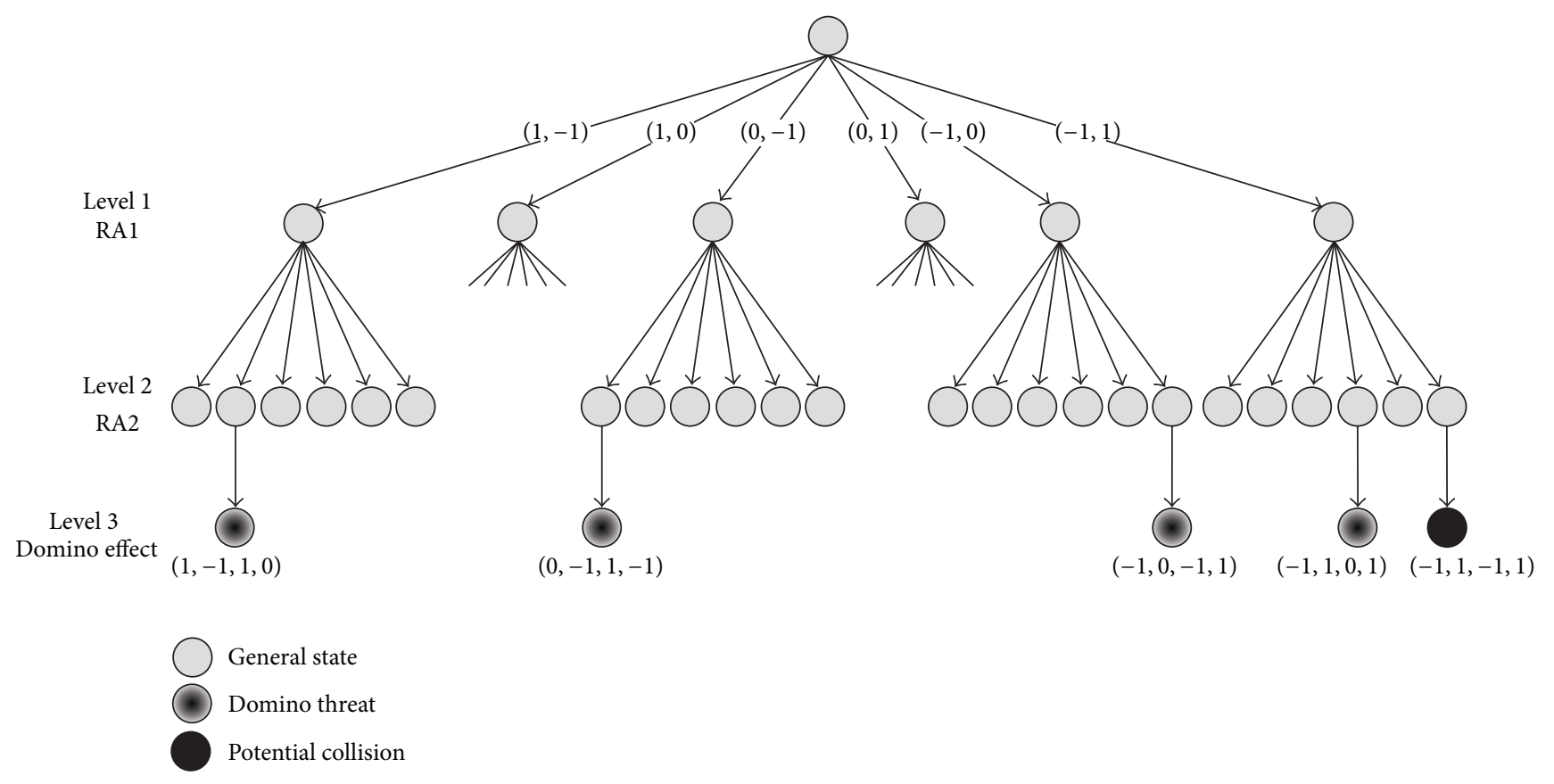

FIGURE 4: The reachability tree of this four-aircraft scenario.

Further investigations into the controller implementation as well as robustness analysis with more realistic uncertainty characteristic are concentrated. Besides, integrating traditional aviation with the Remotely Piloted Aircraft (RPA) to operate in the nonsegregated airspace is also dedicated.

\section{Conflict of Interests}

The authors declare that there is no conflict of interests regarding the publication of this paper.

\section{Acknowledgments}

This research is partially supported by the National Natural Science Foundation of China (no. 61272336) and the Ministry of Economy and Competitiveness in the project "Fire Guided Unmanned Aircrafts and Resources Distribution (FireGUARD)," CICYT Spanish program TIN2011-29494C03-01. Support for Jun Tang from the China Scholarship Council is also acknowledged.

\section{References}

[1] Department of Transportation (DoT), Introduction to TCAS II Version 7.1, Department of Transportation, Federal Aviation Administration, Washington, DC, USA, 2011.

[2] C. Livadas, J. Lygeros, and N. A. Lynch, "High-level modeling and analysis of the traffic alert and collision avoidance system (TCAS)," Proceedings of the IEEE, vol. 88, no. 7, pp. 926-948, 2000.

[3] T. Jun, M. A. Piera, and S. Ruiz, "A causal model to explore the ACAS induced collisions," Proceedings of the Institution of Mechanical Engineers, Part G: Journal of Aerospace Engineering, vol. 228, no. 10, pp. 1735-1748, 2014.
[4] M. J. Kochenderfer, L. P. Espindle, J. K. Kuchar, and J. D. Griffith, "Correlated encounter model for cooperative aircraft in the national airspace system version 1.0," Project Report ATC-344, Lincoln Laboratory, Lexington, Mass, USA, 2008.

[5] F. Netjasov, A. Vidosavljevic, V. Tosic, M. H. C. Everdij, and H. A. P. Blom, "Development, validation and application of stochastically and dynamically coloured Petri net model of ACAS operations for safety assessment purposes," Transportation Research Part C: Emerging Technologies, vol. 33, pp. 167-195, 2013.

[6] E. J. Garcia Gonzalez, Development of a 3-dimensional mathematical collision risk model based on recorded aircraft trajectories to estimate the safety level in high density en-route airspaces [Ph.D. thesis], E.T.S.I. Aeronáuticos (UPM), 2013.

[7] T. B. Billingsley, L. P. Espindle, and J. D. Griffith, "TCAS multiple threat encounter analysis," Project Report ATC-359, Massachusetts Institute of Technology, Lincoln Laboratory, 2009.

[8] T. Jun, M. A. Piera, and J. Nosedal, "Analysis of induced traffic alert and collision avoidance system collisions in unsegregated airspace using a colored petri net model," Simulation, vol. 91, no. 3, pp. 233-248, 2015.

[9] J. Tang, M. A. Piera, and O. T. Baruwa, "A discrete-event modeling approach for the analysis of TCAS-induced collisions with different pilot response times," Proceedings of the Institution of Mechanical Engineers Part G: Journal of Aerospace Engineering, 2015.

[10] J. E. Kuchar and A. C. Drumm, "The traffic alert and collision avoidance system," Lincoln Laboratory Journal, vol. 16, no. 2, pp. 277-296, 2007.

[11] K. Jensen, L. M. Kristensen, and L. Wells, "Coloured Petri Nets and CPN Tools for modelling and validation of concurrent systems," International Journal on Software Tools for Technology Transfer, vol. 9, no. 3-4, pp. 213-254, 2007. 
[12] M. A. Piera and G. Mušič, "Coloured Petri net scheduling models: timed state space exploration shortages," Mathematics and Computers in Simulation, vol. 82, no. 3, pp. 428-441, 2011.

[13] E. Villani, J. C. Pascal, P. E. Miyagi, and R. Valette, "A Petri net-based object-oriented approach for the modelling of hybrid productive systems," Nonlinear Analysis, Theory, Methods and Applications, vol. 62, no. 8, pp. 1394-1418, 2005.

[14] K. Jensen and L. M. Kristensen, Colored Petri Nets: Modelling and Validation of Concurrent Systems, Springer, Berlin, Germany, 2009.

[15] K. Jensen, L. M. Kristensen, and T. Mailund, "The sweep-line state space exploration method," Theoretical Computer Science, vol. 429, pp. 169-179, 2012.

[16] K. Salimifard and M. Wright, "Petri net-based modelling of workflow systems: an overview," European Journal of Operational Research, vol. 134, no. 3, pp. 664-676, 2001.

[17] M. Narciso, M. A. Piera, and A. Guasch, "A methodology for solving logistic optimization problems through simulation," Simulation, vol. 86, no. 5-6, pp. 369-389, 2010.

[18] Eurocontrol, "The EUR RVSM Pre-implementation safety case, version 2.0," Document RVSM 691, 2001, http://dependability.cs .virginia.edu/research/safetycases/EUR_RVSM.pdf.

[19] O. T. Baruwa and M. A. Piera, "Anytime heuristic search for scheduling flexible manufacturing systems: a timed colored Petri net approach," The International Journal of Advanced Manufacturing Technology, vol. 75, no. 1-4, pp. 123-137, 2014.

[20] O. T. Baruwa and M. A. Piera, "Identifying FMS repetitive patterns for efficient search-based scheduling algorithm: a colored Petri net approach," Journal of Manufacturing Systems, vol. 35, pp. 120-135, 2015. 


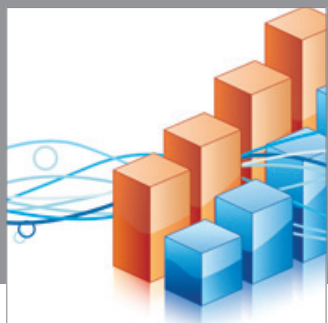

Advances in

Operations Research

mansans

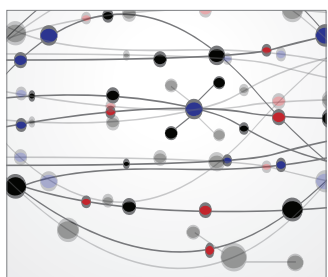

The Scientific World Journal
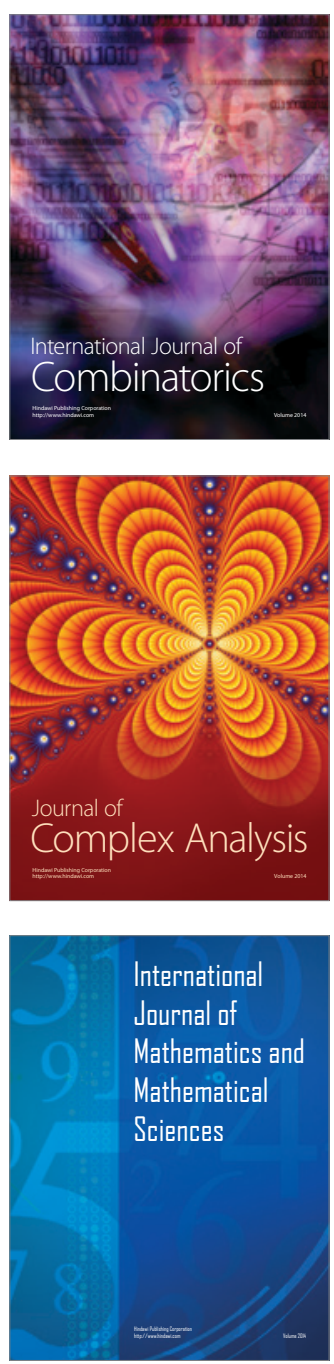
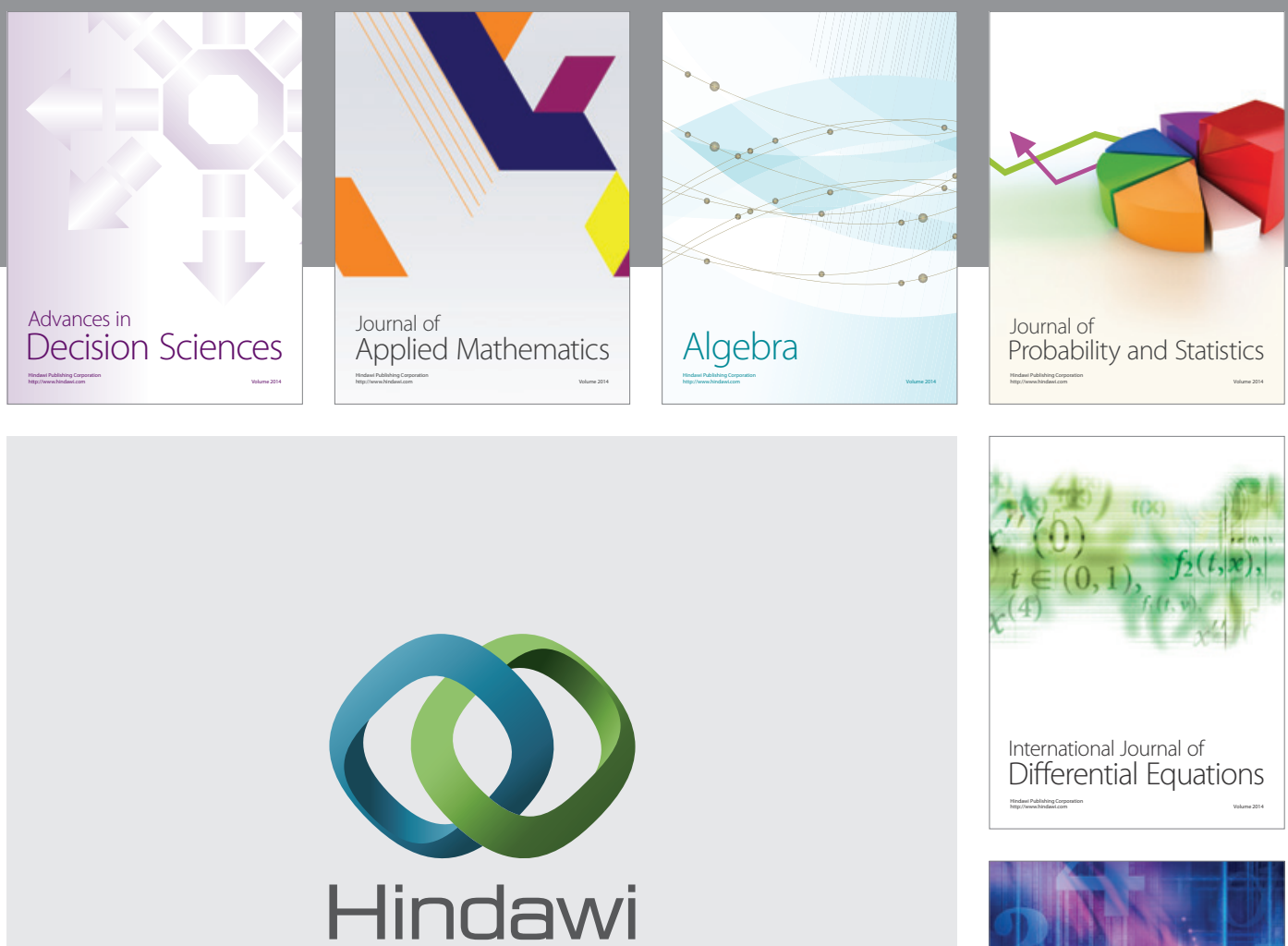

Submit your manuscripts at http://www.hindawi.com
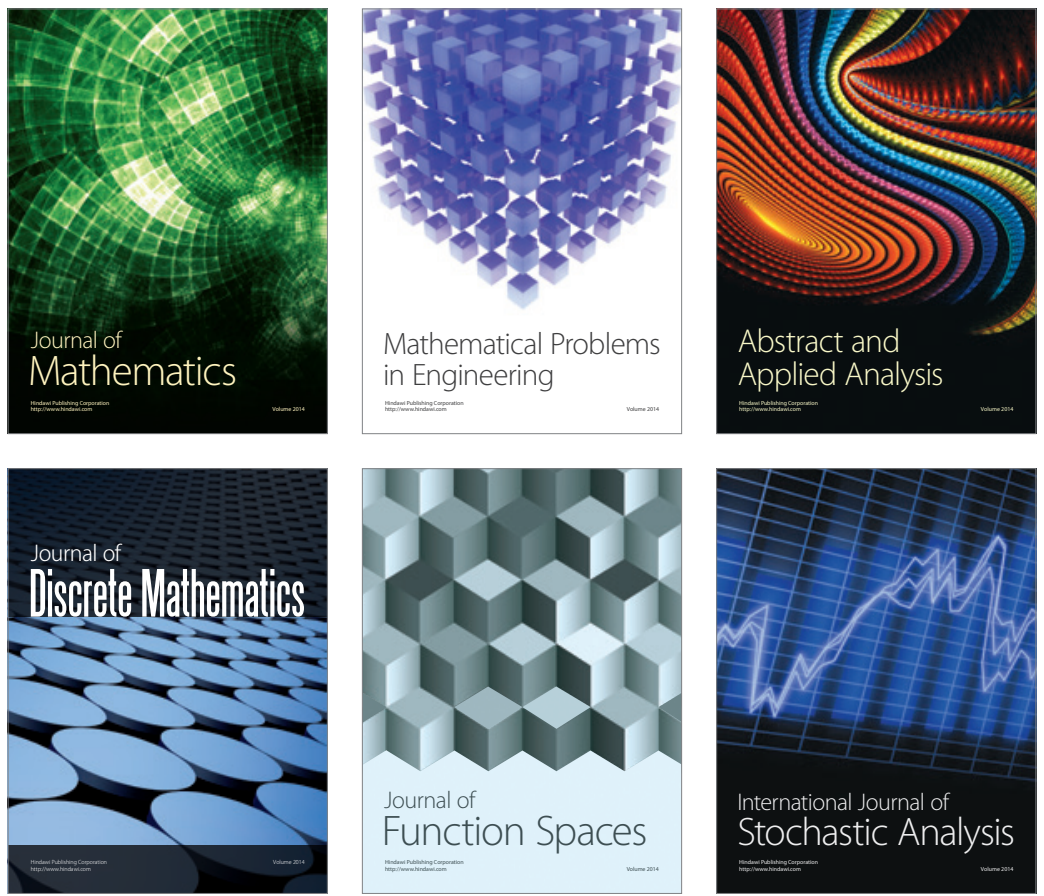

Journal of

Function Spaces

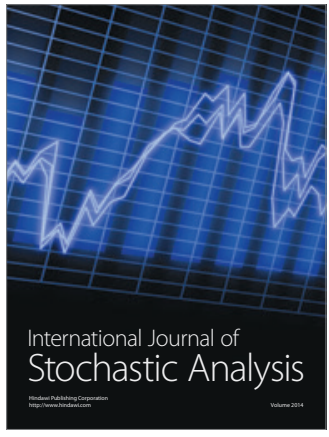

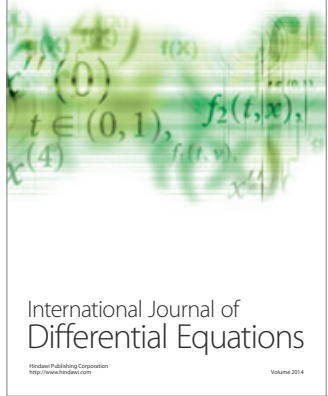
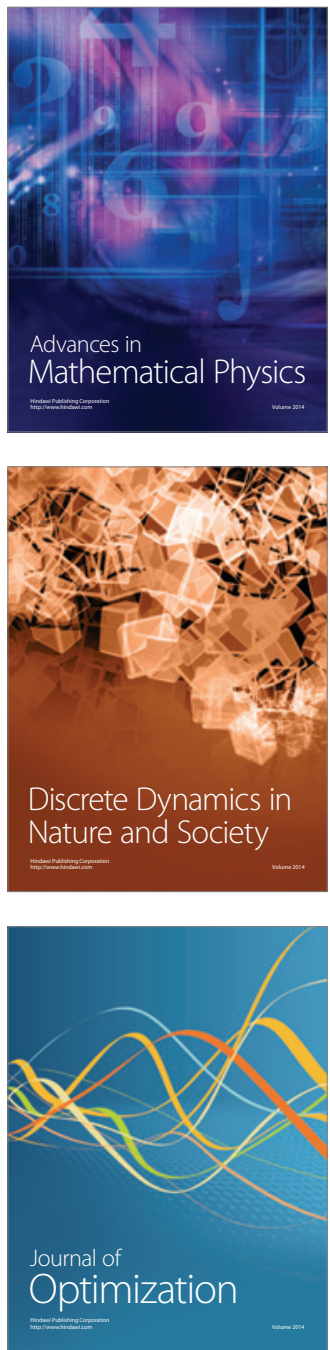